\title{
Archéopages Archéopages
}

Archéologie et société

Le traitement des corps des ennemis et des vaincus. L'exemple du Nord-Est de la France pendant la

\section{Grande Guerre}

\section{Guy Flucher}

\section{(2) OpenEdition}

\section{Journals}

Édition électronique

URL : https://journals.openedition.org/archeopages/545

DOI : 10.4000/archeopages.545

ISSN : 2269-9872

Éditeur

INRAP - Institut national de recherches archéologiques préventives

Édition imprimée

Date de publication : 1 septembre 2014

Pagination : 46-55

ISSN : $1622-8545$

\section{Référence électronique}

Guy Flucher, «Le traitement des corps des ennemis et des vaincus. L'exemple du Nord-Est de la France pendant la Grande Guerre », Archéopages [En ligne], 39 | 10/2013-01/2014, mis en ligne le 01 janvier 2016, consulté le 21 janvier 2022. URL : http://journals.openedition.org/archeopages/545 ; DOI : https://doi.org/10.4000/archeopages.545 


\section{Le traitement des corps des ennemis et des vaincus L'exemple du Nord-Est de la France pendant la Grande Guerre}

Guy Flucher Inrap, UMR 7041, ArScAn

\section{6}

1. Cet article synthétise une réflexion de l'auteur présentée dans Flucher 201
Que faire du cadavre de l'ennemi ? Les historiens Luc Capdevila et Danièle Voldman distinguent trois types de comportements - respect, acharnement, anéantissement - qui ne correspondent ni à des périodes chronologiques déterminées ni à des aires géographiques particulières (Capdevila, Voldman, 2002). On peut en ajouter un quatrième, l'indifférence, conséquence de la réification du cadavre suscitée par la mort de masse. Le conflit intervient, en outre, après une phase d'évolution des pratiques funéraires qui s'est déroulée au cours du XIX ${ }^{\mathrm{e}}$ siècle où l'on a vu se développer les cimetières extra-muros et la généralisation des sépultures individuelles à longues durées de concession (Urbain, 1998). En ce qui concerne les tués de la guerre, le conflit francoallemand de 1870-1871 a fondé les bases d'une prise en charge des sépultures de combattants par les États.

À la veille de la Grande Guerre, les traités internationaux recommandent que les corps des ennemis fassent l'objet d'une identification et d'une sépulture entretenue. Ces règles n'ont pas toujours été suivies. Les modalités de transgression ont été variées en fonction des conceptions qu'avaient les belligérants de la guerre en général et du soldat ennemi en particulier. Pour étudier ces comportements, il faut auparavant présenter le cadre général des pratiques funéraires durant le conflit.

Afin d'alléger cette synthèse déjà complexe des pratiques funéraires de la Grande Guerre, nous nous cantonnerons à deux belligérants aux antagonismes militaro-culturels marqués, les Français et les Allemands'.

\section{Les sources}

La Première Guerre mondiale a généré une abondance de morts mais aussi de sources. Avant celui-ci, aucun conflit n'en a généré autant. Bureaucratie militaire, alphabétisation massive, démocratisation de la photographie, développement des services postaux, amplitude de la mobilisation, désir de témoigner de la part des combattants, autant de facteurs qui ont contribué à produire du document. En raison du caractère général de la mobilisation des populations civiles, la question de la destination ultime des victimes du conflit est vite devenue prégnante. L'administration militaire s'est adaptée en créant des services d'état-civil aux armées chargés de localiser au mieux l'emplacement des sépultures et de veiller à l'identité des défunts. Ainsi, ce sont des milliers de plans, de croquis et de registres qui sont disponibles aux chercheurs contemporains (Flucher, 2011).

Le développement de l'archéologie préventive dans les zones de l'ancien front a généré la mise au jour de nombreuses traces du conflit. Parmi elles, les corps de combattants tiennent une place importante. Il ne s'agit pas toujours de sépultures, dans la mesure où de nombreux corps ont été enfouis accidentellement. Il appartient alors à l'anthropologue de faire la distinction entre l'enfouissement fortuit, l'inhumation sommaire et l'inhumation associée à un ensemble de gestes rappelant ceux de la vie civile en temps de paix ou propres aux usages militaires (Desfossés, Jacques, Prilaux, 2008).

\section{La sépulture idéale}

Les témoignages ainsi que l'évolution de la réglementation au cours du conflit indiquent clairement que pour les combattants et leurs familles, la sépulture idéale consiste en une inhumation individuelle en cercueil au sein d'un espace funéraire particulier, le cimetière, avec un souci affirmé de préserver l'identité du défunt par une signalisation de surface. Un corps, un nom, un lieu, voilà ce qui résume la volonté commune et qui contraste avec 


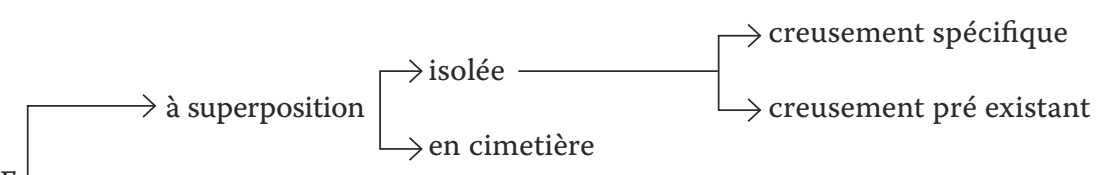

2

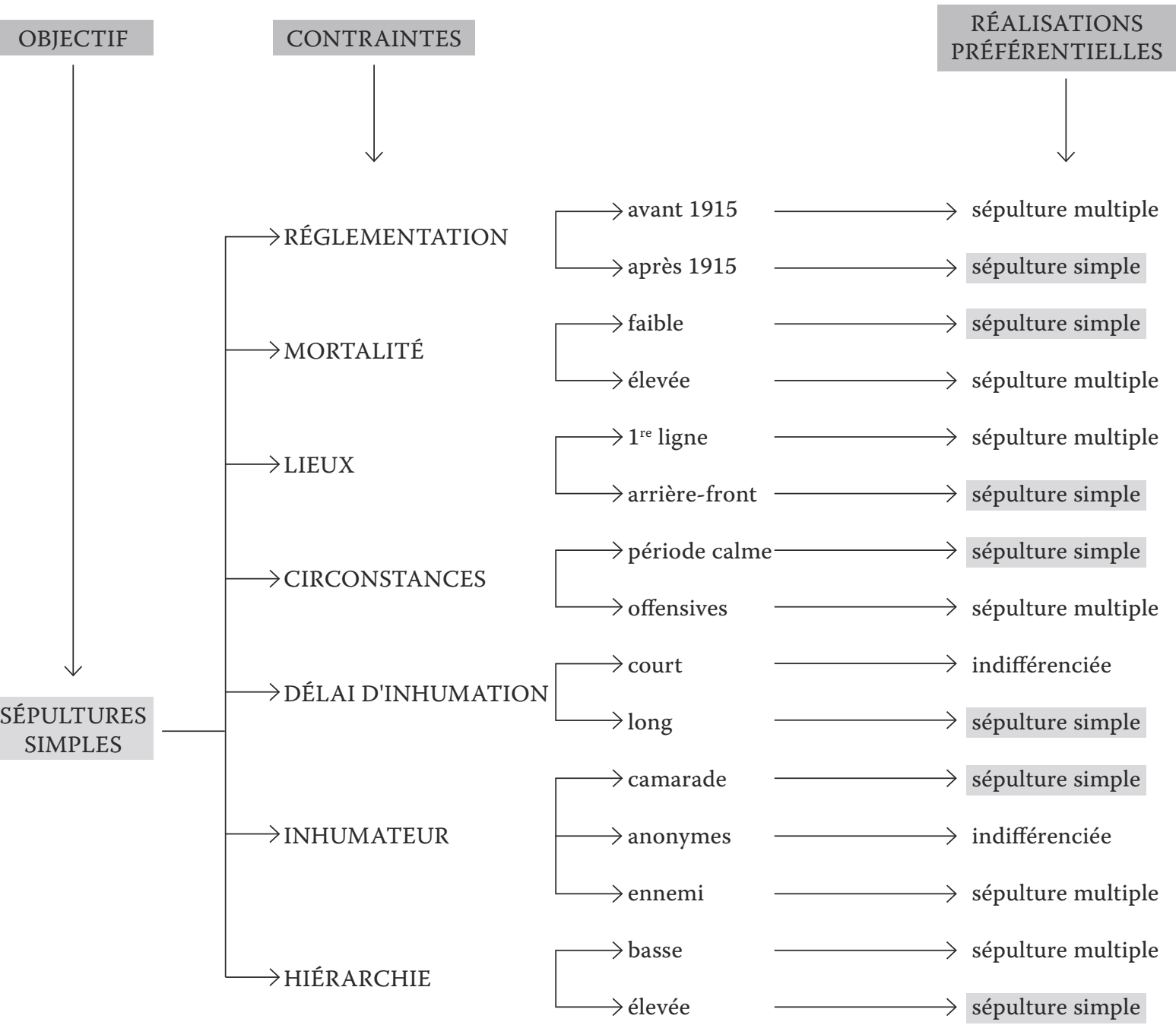




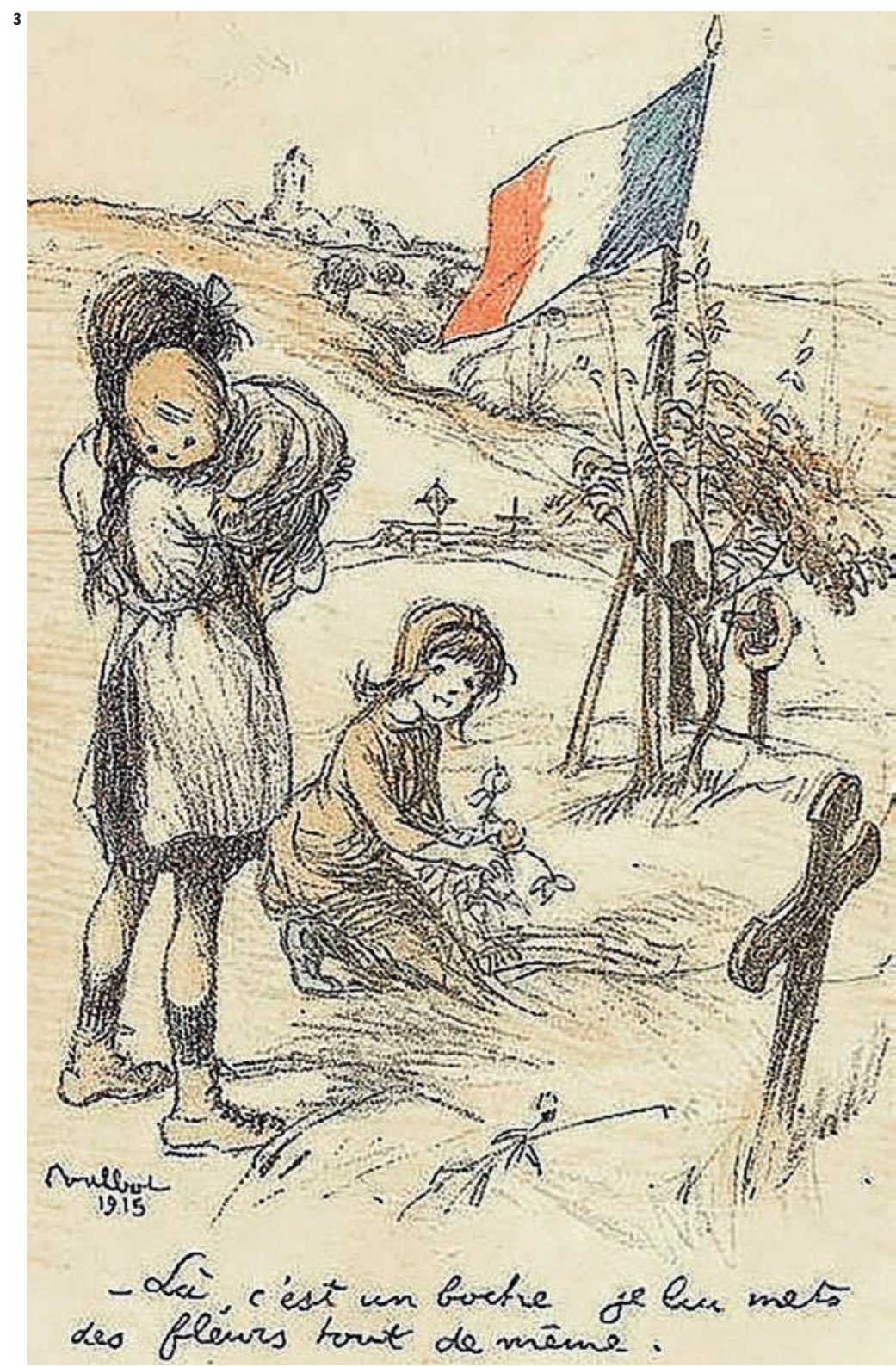

3. Carte postale de

propagande française. « Là

c'est un boche, je lui mets des

fleurs tout de même n, dessin de Poulbot, 1915.

4. Carte postale de

propagande allemande. « Hier

ruhen 3 tapferer franz.

Kriegen - Ci gisent 3

valeureux soldats francais 1 .

La question du respect

manifesté aux ennemis morts

est un sujet de propagande

tant francaise qu'allemande.

S'il s'agit de montrer le

respect des conventions

internationales, il est

possible que cette volonté

de propagande ait influencé

la réalité des pratiques.

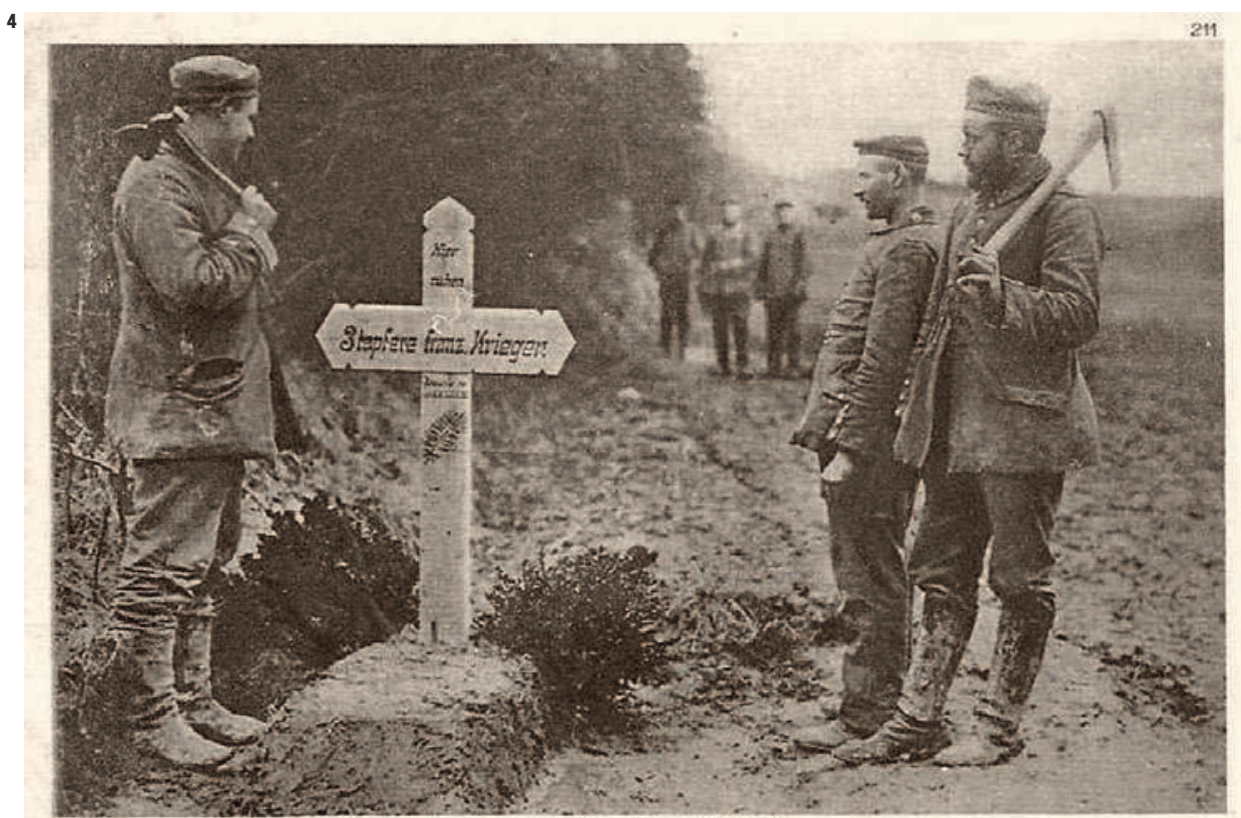

DEUT SCHES GRABKREUZ FÜR GEFALLENE FRANZOSEN. 
les pratiques usuelles dans les contextes de mort de masse (Signoli, 2008).

Les pratiques montrent que ces vœux n'ont pas été pleinement réalisés. Pour les comprendre, il faut les mettre en relation avec la temporalité et la topographie du conflit. Il est clair que l'on n'inhume pas de la même façon en août 1914 qu'en novembre 1918. De même faut-il distinguer les périodes de guerre de mouvement de celles de position ainsi que ce qui se passe en première ligne sous la mitraille et à l'arrière-front autour des centres de soins. Cette diversité des temps et des lieux d'inhumation a engendré en parallèle une grande variabilité de pratiques [ill. 1]. Durant les premiers mois de la guerre, les inhumations en sépultures multiples (dites « fosses communes » dans la terminologie militaire) sont fréquentes. Avec la stabilisation du front et la mise en place de procédures adaptées, les cadavres sont plus souvent transportés un peu à l'arrière afin d'être inhumés en sépultures individuelles. Dans les situations d'offensives meurtrières, où le transport des corps devient très dangereux pour les brancardiers, les inhumations peuvent être réalisées à la hâte en utilisant des creusements générés par le conflit (trous d'obus, tranchées désaffectées). La mort de masse a connu des pics d'intensité au cours desquels l'inhumation en sépulture individuelle devenait difficile à réaliser malgré les bonnes volontés et les directives générales concernant les tués de la guerre. Aussi a-t-on vu se développer au cours du conflit un type de sépulture multiple particulier, la sépulture en tranchée. Le principe consiste à inhumer plusieurs corps dans un seul creusement (généralement une tranchée de $2 \mathrm{~m}$ de large sur plusieurs dizaines de mètres de long) mais sans les superposer. À chaque corps correspond néanmoins en surface une croix nominative afin que l'individualité et l'identité du défunt soit respectée.

La seule décision d'une inhumation en sépulture simple ou multiple (en faisant abstraction de la question des cercueils ou du cimetière) est le fruit d'une quantité de facteurs [ill. 2]. Ainsi, un soldat de la Grande Guerre avait toutes les chances de bénéficier d'une inhumation en sépulture individuelle s'il était gradé, décédé après 1915 dans la zone de l'arrière-front en période calme et s'il était enterré par des camarades. À l'inverse, il avait tous les risques d'être inhumé en sépulture multiple s'il était troupier, décédé avant 1915 en première ligne lors d'une offensive meurtrière et s'il était enterré par un ennemi. Entre ces deux extrêmes, une quantité de situations sont envisageables, qu'il n'est pas encore possible de quantifier. On comprend donc que s'interroger sur le traitement des corps des ennemis dans ce contexte oblige à ne comparer que ce qui est comparable. La mise en vis-à-vis de comportements selon les nationalités n'a de sens que dans une même unité de temps et de lieu.

\section{Le corps de l'ennemi}

Comment, sur le terrain, va-t-on enterrer le « Boche », le « Franzouse» ? La question est plus importante qu'il n'y paraît. Le respect des morts « de l'autre » ou « par l'autre » ainsi que les pratiques transgressives constituent un thème de propagande usité durant le conflit, avec rappel des conventions internationales et accusations réciproques de «barbarie » [ill. 3 et 4]. À cette frontière floue entre réalité, rumeur et propagande, il est nécessaire de faire un point sur la question de la crémation de masse. En juin 1915, des députés français ont déposé un projet de loi à la Chambre demandant la crémation des cadavres ennemis. Cette loi a été repoussée par le Sénat à la suite de rapports présentés par le sénateur Cazeneuve au nom de la commission de l'Armée. Le même sénateur avait par ailleurs affirmé que les Allemands, eux, pour se débarrasser des cadavres, les faisaient transporter par voie ferrée dans des wagons et les incinéraient dans les hauts fourneaux de la région du Nord (Journal officiel de la République française, débats parlementaires, Sénat, janvier 1916, cité par Capdevila, Voldman, 2002, p. 161). La crémation serait donc, en théorie, réservée aux cadavres des ennemis ou pratiquée par eux. Dans les faits qu'en était-il ? D'un point de vue législatif, la crémation des combattants n'a pas été autorisée. Sur le terrain, cependant, quelques rares témoignages évoquent cette question. Louis Barthas écrit ainsi :

"Au coin d'une petite place, en me promenant, japerçus un attroupement. J'y courus et ne vis d'abord rien de très curieux: on faisait cercle autour d'un bloc brunâtre, graisseux, avec des morceaux d'os très apparents. Qu'était-ce donc cela? On m'expliqua qu'en août 1914, à la suite des terribles combats qui se livrèrent dans la région, les Allemands maîtres du champ de bataille ramassèrent les morts - les leurs et les nôtres - et en firent un immense bûcher qu'ils arrosèrent de pétrole et pendant plusieurs jours le vent apporta à plusieurs lieues à la ronde cette épouvantable odeur de chair grillée. Venu de ce bûcher on ne savait comment, ce bloc que j'avais devant les yeux était un morceau de ce qui était resté lorsque le sinistre foyer séteignit. ». (Barthas, 1987, p. 269).

Mais, dans la plupart des cas, les auteurs n'ont pas assisté de leurs yeux à ces actes. Il est possible que la crémation de masse ait été pratiquée, mais dans le contexte particulier et circonscrit dans le temps des hécatombes de l'été et de l'automne 1914. Si de tels actes ont eu lieu, il faut les comprendre comme une mesure d'assainissement du champ de bataille qui n'a pas forcément de rapport avec le manque de considération pour les corps de l'ennemi.

On ne sera pas étonné de constater qu'il n'y a pas forcément adéquation entre les directives générales de l'administration concernant les sépultures des ennemis et les usages sur le terrain. Pour comprendre ces derniers, il faut se rapprocher au plus près de la réalité. Qui inhume? Le plus 


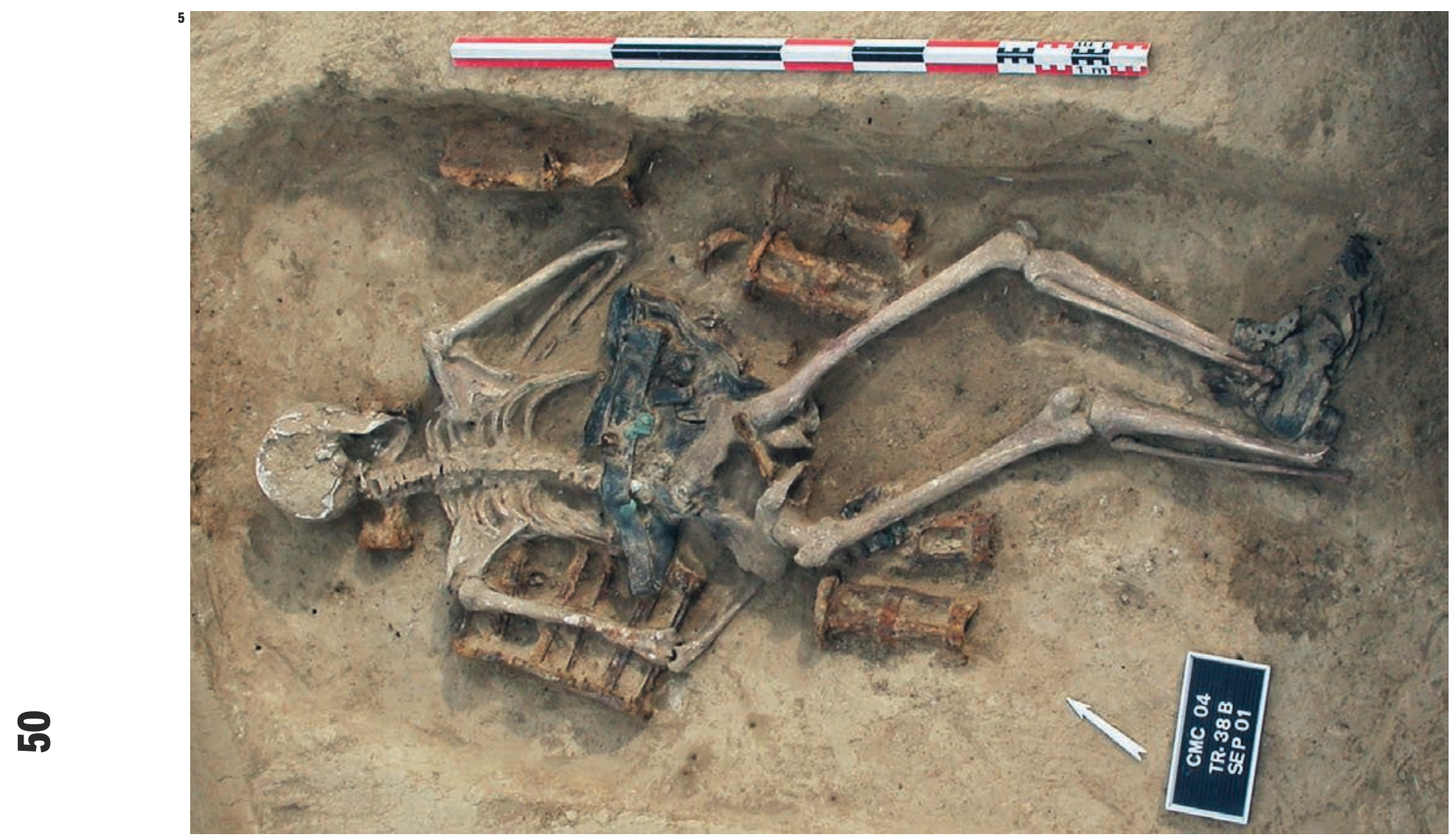

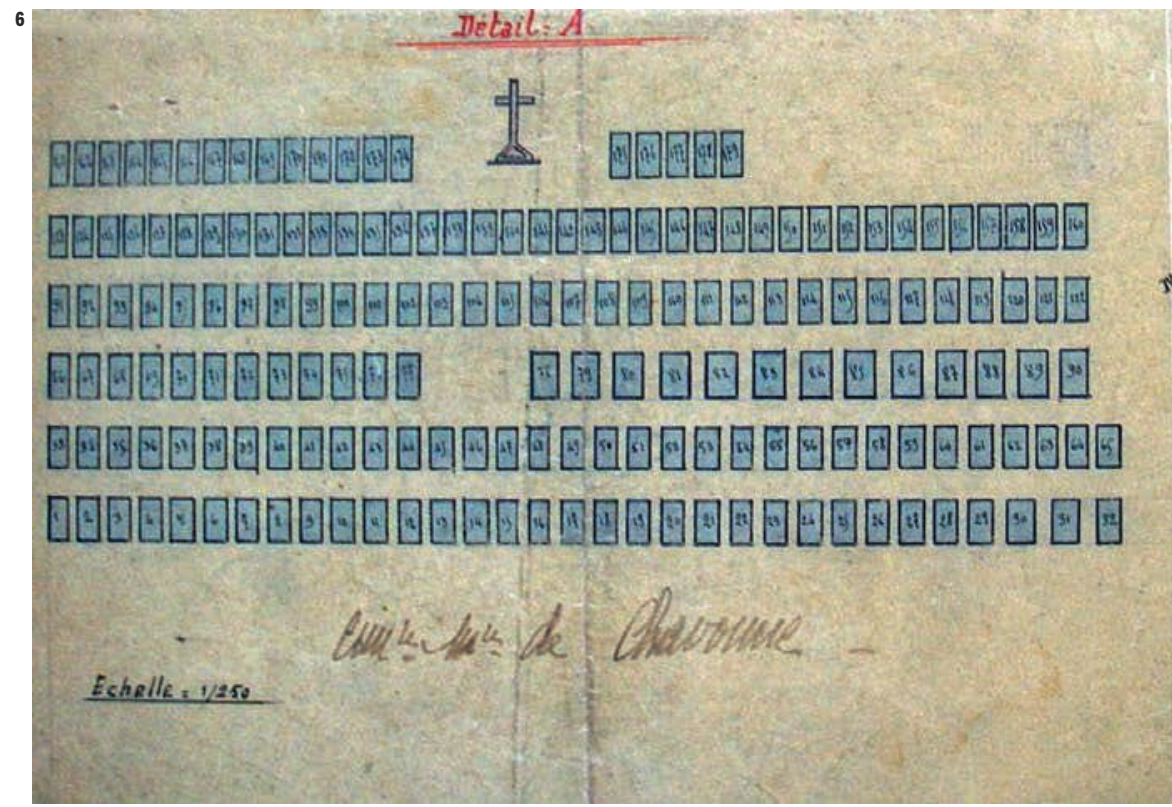

5. 5. À Courmelles (Aisne), un diagnostic (mené par Sylvain Thouvenot, Inrap, au « Mont de Coumelles " en 2004), a mis au jour cette inhumation sur le ventre d'un soldat allemand.

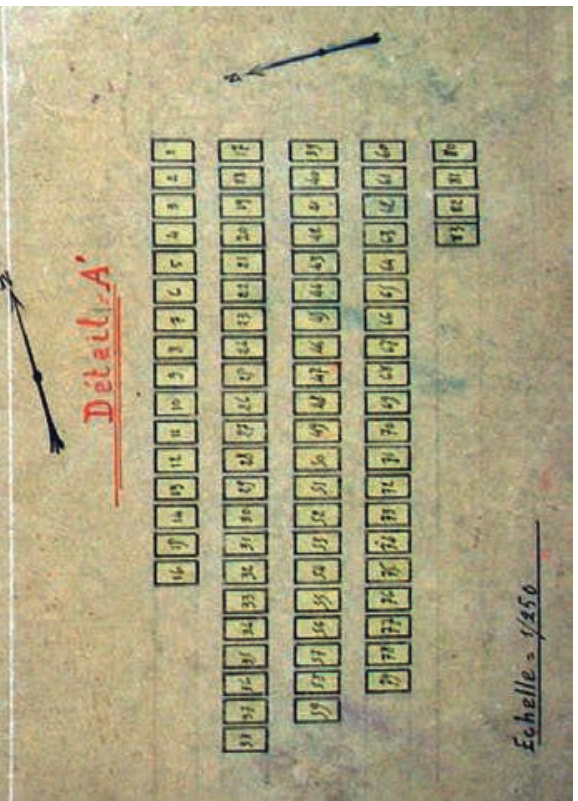

6. Sur ce plan du cimetière militaire français de Chavonne (Aisne), où les sépultures allemandes ont été figurées en jaune, la volonté

de distinguer les tombes des soldats ennemis s'illustre par l'inhumation avec z une orientation différente. 
souvent, il s'agit d'hommes mobilisés dans les groupes de brancardiers divisionnaires. Dans la vie civile, beaucoup d'entre eux exerçaient la profession de musicien (Retailleau, 2003) ou étaient servants de cultes religieux (Pousse, 200o). Il sont secondés dans leur tâche par des régiments de territoriaux, composés d'hommes âgés dispensés en partie des combats. Fait important à mentionner ici, les prisonniers de guerre, avant d'être envoyés dans des camps à l'arrière, ont parfois participé aux inhumations comme l'attestent de nombreux témoignages et photographies (Cazals, 20o8, p. 78-79). Cette réalité permet de relativiser d'éventuels discours sur les sentiments de haine entre les belligérants. Sur le terrain, il y a des hommes qui partagent les mêmes souffrances et la pénible tâche d'inhumer les victimes du conflit.

Les fouilles archéologiques ont donné l'occasion de découvrir des sépultures de combattants et de mettre en évidence des gestes funéraires peu ou pas décrits dans les témoignages et les comptesrendus. Par exemple, des soldats français comme allemands ont été découverts inhumés en sépulture individuelle, sur le ventre, face contre terre [ill. 5]. Il serait trop facile et superficiel d'en proposer comme interprétation une manifestation d'opprobre envers le cadavre d'un ennemi. Pour ce faire, il faudrait en premier lieu connaitre l'identité du défunt afin de savoir quand il est tombé et par conséquent par qui il a été inhumé en fonction des aléas des mouvements de troupes lors des offensives. Dans la plupart des cas, il est impossible d'avoir accès à la totalité de ces données, malgré l'abondance des sources. Par contre, il arrive qu'on puisse cerner au mieux les circonstances du décès et de l'inhumation à partir d'informations fragmentaires comme le type de régiment, en examinant les vestiges d'uniformes. De là, la lecture des archives des brancardiers divisionnaires peut nous indiquer qu'à tel endroit, à tel moment, il s'est passé dix jours entre le décès et l'inhumation et que les corps ont été basculés dans les fosses au moyen de crocs à longs manches en raison de leur état de décomposition rendant la manipulation trop pénible. Deux interprétations possibles pour un fait, une inhumation sur le ventre, dont l'une est étayée par les réalités concrètes et parfois cruelles du traitement des corps au cours de ce conflit, quelles que soient les nationalités concernées.

La haine qui peut habiter les hommes en guerre a parfois conduit à des pratiques d'acharnement envers le cadavre de l'ennemi, jusqu'à son anéantissement. Depuis que les conflits existent, nombreux sont les exemples de violences exercées envers les cadavres, comme les démembrements ou la décomposition en place publique. Il ne semble pas que de tels comportements aient eu lieu durant la Grande Guerre. Certes, quelques témoignages font état de mutilations de cadavres, mais leur motivation était principalement la récupération de bijoux et de dents en or. La vénalité plus que la haine a été à l'origine de ces actes. Il n'est pas interdit de penser que l'extrême violence faite aux corps par les armes modernes suffisait à faire perdre sa superbe au corps de l'ennemi. Pour autant, les pratiques discriminantes n'ont pas été absentes. Elles ont eu trait non pas à l'intégrité physique du défunt, mais au respect de son identité et à la place qu'on lui a accordé dans les espaces funéraires. Comme nous l'avons vu, les mentalités ont évolué au cours du XIX ${ }^{\mathrm{e}}$ siècle dans le sens de la généralisation de la sépulture individuelle, de la longévité des concessions et de la pérennisation de l'identité des morts. C'est sur ces registres que vont principalement se jouer les discriminations.

\section{Préserver l'identité}

Un corps, un lieu, un nom. De nombreux combattants ont établis des relations avec les familles de proches tombés au combat afin de les informer sur les événements ayant occasionné la mort et surtout sur le lieu de sépulture. Ce type de démarche, fréquent dans chaque camp, semble avoir été rarissime vis-à-vis de l'ennemi. On peut cependant citer deux témoignages, l'un d'un infirmier français : "Derrière Pr... j’ai trouvé et enseveli un cadavre de Boche, dont jai recherché et découvert les papiers dans l'intention de les faire parvenir à sa famille. Pouvez-vous les faire parvenir par le bureau de Genève? " (Pottecher, 2003, p. 85); l'autre d'un sous-lieutenant allemand: "J'avais l'intention de faire enterrer le lieutenant Stokes derrière ma tranchée... Le bataillon réclama le corps pour le faire ensevelir à l'arrière. Je suis donc débarrassé de ce devoir de camaraderie. Une fois la guerre finie, jécrirai peut-être encore à la famille de ce brave officier pour l'informer sur son sort et sur sa sépulture » (Jünger, 2014, p. 300-301). Ces deux exemples ne sont assurément pas représentatifs. Le premier émane d'un jeune pacifiste engagé volontaire comme secouriste, tandis que le second est celui d'un jeune intellectuel pétri d'idéaux chevaleresques.

Néanmoins, l'étude des archives des services d'état-civil des armées montre que, de façon générale, il n'y a pas une volonté délibérée d'omettre de mentionner l'identité de l'ennemi défunt dans les registres comme sur les signalisations de surface. Peut-être faut-il y voir un respect réciproque des conventions internationales en la matière, d'autant que la mauvaise gestion des tués de la guerre de 1870-1871 était restée dans les mémoires. Il faut garder aussi à l'esprit le fait qu'avec les aléas du conflit et les déplacements des zones de combats, chacun des belligérants pouvait avoir l'opportunité de constater de visu comment l'ennemi traitait les sépultures de leurs compatriotes et d'en tirer profit en terme de propagande.

\section{Sépulture simple ou multiple pour l'ennemi}

Comme nous l'avons vu précédemment, cette distinction dans les modes d'inhumation est à mettre en relation avec les phases et les formes 

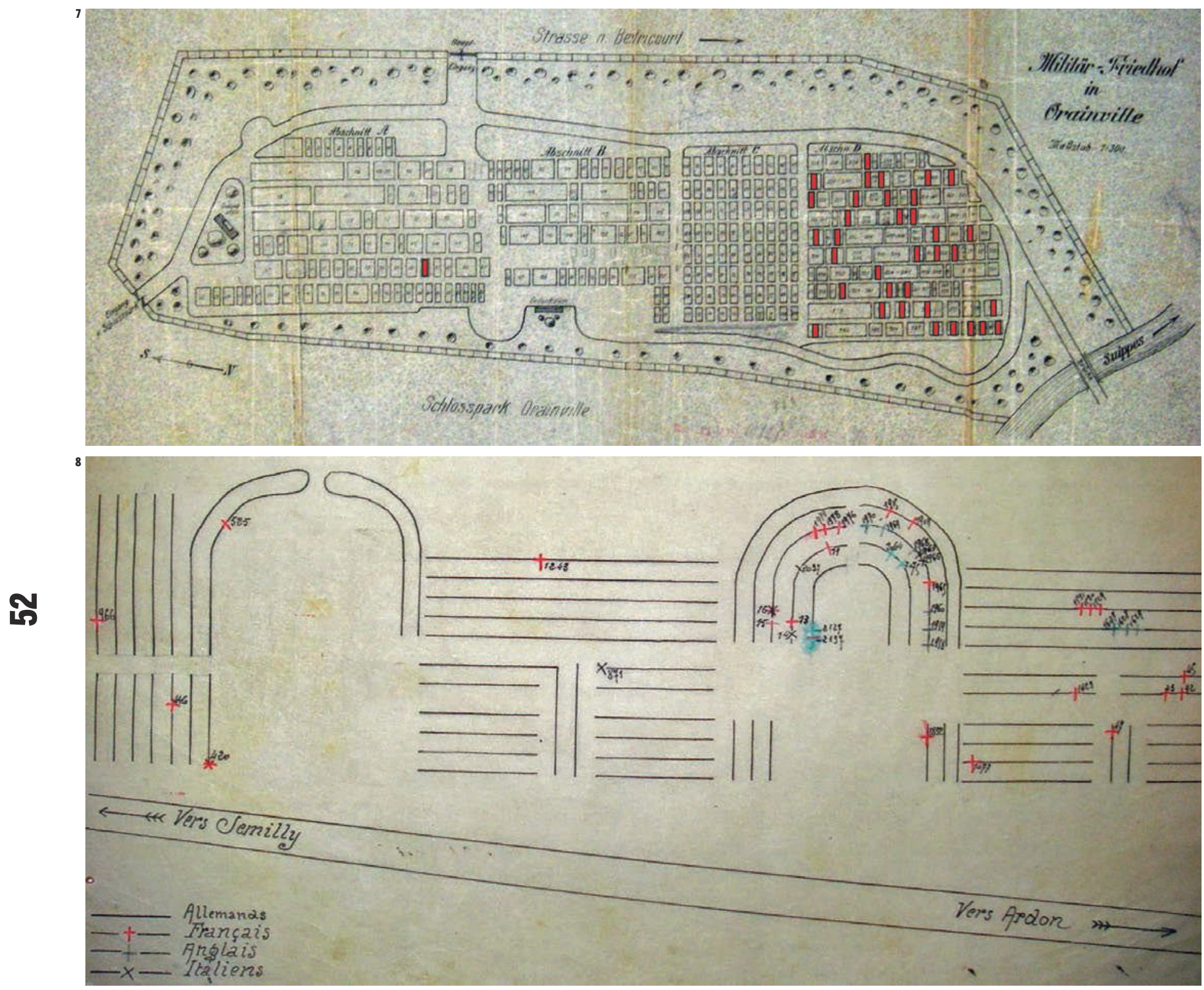

7. et 8. Dans les

cimetières militaires

allemands, la solution

choisie a souvent été

l'absence de distinction

en fonction des nationalités,

comme le montrent

le plan du cimetière

d'Orainville, Aisne (en

haut) où les sépultures

françaises figurent en

rouge, et celui du

cimetière de Laon-

Bousson, Aisne (en bas)

où Français, Anglais

et Italiens sont intégrés. 
du conflit. À la suite des hécatombes de 1914, l'usage de la « fosse commune » a été fréquent chez les deux belligérants et on ne distingue pas de discrimination de pratique selon l'origine des défunts. Cependant, on voit émerger dès le début du conflit des différences de comportement entre Français et Allemands. Les premiers, dans l'aménagement des «fosses communes », s'attachent fréquemment à ne pas mélanger les corps de nationalités ennemies. À l'inverse, les Allemands ont plutôt tendance à réunir les corps sans distinction d'origine, pourvu qu'ils soient morts au combat, comme le montrent les dédicaces des monuments funéraires ${ }^{2}$.

Par la suite, l'examen de cette question est rendue difficile en raison de la nature des sources. La fosse commune à empilement de corps tend à disparaître au profit des sépultures multiples en tranchée. Or, la façon dont elles sont figurées sur les plans et leur aspect de surface ne permettent pas toujours de les distinguer des sépultures simples organisées en rangées serrées. Néanmoins, une partie des plans réalisés par les services d'étatcivil des armées utilisent des codes graphiques permettant de distinguer les «fosses communes » et des codes de couleur pour les nationalités. Ainsi, on peut mettre évidence le fait que les Français, dans une même unité de temps et de lieu, ont tendance à inhumer en sépulture multiple leurs ennemis un peu plus souvent que leur compatriotes.

\section{Les espaces funéraires}

Pour le combattant de la Grande Guerre, pouvoir reposer au cimetière est une préoccupation non négligeable. De nombreux témoins font état de ce désir, pour eux-mêmes comme pour leurs camarades, dans le souci du respect du deuil des familles. Ferdinand-Antonin Vuillermet l'explique :

«Certains sétonnent que nous nous occupions ainsi de nos morts et que nous ne les enterrions pas sur le champ de bataille où ils sont tombés. C'est l'habitude de notre division. Quand les circonstances nous le permettent, - et nous faisons l'impossible pour qu'il en soit toujours ainsi - nous voulons que les morts de nos bataillons dorment tranquilles de leur dernier sommeil, tous réunis autour de la croix d'un cimetière paisible. Tous auront une fosse individuelle et ceux qui ne pourront pas avoir de cercueil seront pieusement ensevelis dans une toile de tente. Les familles de nos chasseurs ne trouveront-elles pas un adoucissement à leur douleur, dans la certitude que des camarades ont fait pour leurs enfants ce qu'elles auraient si vivement désiré faire elles-mêmes? Nous voulons qu'un jour on puisse venir prier sur ces tombes. " (Vuillermet, 1918, p. 189). Avec la mise en place des services de santé à l'arrière-front, de nouveaux espaces funéraires spécifiquement militaires ont été créés ex nihilo. Ces cimetières, aménagés à l'abri des combats les plus violents, sont moins soumis à l'urgence de l'inhumation de masse. Dans ces lieux, il y a le temps nécessaire de la réflexion quant à la destination du cadavre de l'ennemi.

Les plans et les registres associés nous permettent d'observer la façon dont ont été disposées spatialement les sépultures des ennemis. Trois principaux cas de figure se présentent : l'intégration, la relégation et la spécification de l'espace. Côté français, dans les situations où les corps allemands à enterrer ont été peu nombreux, voire en nombre presque anecdotique, ils ont souvent été intégrés au sein des rangées de soldats de nationalité française. Cependant, dans certains cas, cette intégration n'a pas eu lieu et les ennemis à inhumer (même en faible nombre) ont été relégués dans un espace éloigné du cimetière ou plus radicalement à l'extérieur de la clôture de celui-ci [ouverture p. 4-5]. On remarque aussi, parfois, des distinctions dans l'orientation des sépultures pour mieux différencier les nationalités entre elles. Dans les situations de forte mortalité, la pratique la plus fréquente a été la création d'un espace funéraire spécifique pour les morts allemands [ill. 6]. Les tendances qui se manifestent dans la façon dont les Français ont enterré les Allemands vont globalement dans le sens d'une distinction assez nette entre les belligérants au sein des ensembles funéraires. Pour autant, peut-on en conclure à une animosité envers les soldats ennemis qui se traduirait par les places spécifiques qui leur sont accordées dans les cimetières? Comment faire la part des intentions entre une mise à l'écart signifiant l'opprobre ou à l'inverse un respect des identités nationales?

A contrario, la place de la sépulture du soldat ennemi a été envisagée de façon claire par les Allemands. C'est leur intégration dans des ensembles funéraires multinationaux qui a été majoritairement appliquée [ill. 7 et 8]. À ce jour, nous n'avons pas trouvé de traces significatives de relégation (du moins pour ceux « morts au champ d'honneur ») ou de création de cimetières spécifiques pour les soldats français. Cette conception de l'espace funéraire comme lieu de repos commun à tous les combattants, alliés ou ennemis, peut être confirmée par l'étude des nouveaux lieux d'inhumation engendrés par la reprise des grandes offensives du printemps 1918. Plutôt que de créer des petits cimetières isolés dans le cadre de ce nouvel épisode de guerre de mouvement, les Allemands ont fait le choix d'inhumer une partie de leurs morts dans les cimetières militaires français ou dans les cimetières communaux quand il y restait de la place (Service départemental des sépultures de guerre de l'Aisne, dossiers Chassemy et Cys-laCommune). Il semble donc que les Allemands ont considéré avec un égal respect les tués de la guerre, quelle qu'ait été leur nationalité. On doit cependant s'interroger sur les motivations qui ont présidé à ce choix d'égalité de traitement. Dans la mesure où l'armée allemande occupait une partie de territoire où vivaient encore des Français, on peut se demander s'il n'y avait pas là aussi une volonté deutscher und

de héros français et

allemands), inscrip
sur le monument

Condé-sur-Aisne.

"Hier ruhen in Gott 38

französischer soldaten gef

22-8-1914 ", inscription

sur la croix de la
sépulture multiple

Roselles. (Ici reposent en

Dieu 38 soldats allemands

et 20 soldats français

tombés le 22-8-1914). 
de s'attirer en retour une opinion plus favorable de la part des populations civiles.

\section{Rendre hommage}

Contrairement aux Français, les Allemands ont érigés de façon presque systématique des monuments funéraires collectifs dans leurs cimetières militaires. Certaines de leurs dédicaces rendent hommage (en allemand) aux soldats ennemis : "Freund und Feind im Tod vereint» («Amis et ennemis unis dans la mort », monument de Dieuze, Moselle). Dans certains cas, la dédicace est traduite en français : «À la mémoire des soldats de la XIII ${ }^{\mathrm{e}}$ division de réserve allemande et des soldats du XVIII ${ }^{\mathrm{e}}$ corps d'armée français morts pour leur patrie » (Monument allemand de Pancy-Courtecon, Aisne). Cette considération envers l'ennemi mort au combat peut aussi s'exprimer par des inscriptions sur les croix de bois [ill. 9 et 4]. Quantitativement, ces hommages ne sont pas légion et on ne peut exclure une intention de propagande.

\section{Quand l'ennemi devient le vaincu}

Par le traité de Versailles de 1919, l'Allemagne reconnaît sa défaite. Les corps des soldats allemands tombés sur le territoire français deviennent alors des corps de vaincus. Par ce même traité, l'entretien des sépultures allemandes passe sous la responsabilité des autorités françaises. Des millions de sépultures et des milliers de cimetières sont disséminés sur le territoire. La remise en état des zones de l'ancien front, la renaissance des villages et de l'agriculture sont confrontées à la présence gênante et envahissante des tués de la guerre. Ainsi, la reconstruction du pays passe d'abord par un nettoyage des champs de bataille qui, de fait, aboutit au regroupement des morts dans de nouveaux espaces, appelés dorénavant nécropoles nationales. Ainsi, des millions de corps ont été exhumés à partir de 1919 (Jeauffret, 1997). L’Allemagne n'étant pas autorisée à superviser ces opérations, de multiples indices montrent que les sépultures de leurs soldats ont fait l'objet d'une moindre attention. Ainsi, un rapport de gendarmerie (Archives nationales, F7-12936, département de la Marne, cité par Pourcher, 1993) consigne ce témoignage : "Lorsque j’ai envoyé mon charretier Cheron labourer le champ, je n'ai pas pensé de lui dire de respecter la tombe qui s'y trouvait. À ce moment-là, j’avais entendu dire que cette tombe ne contenait qu'un soldat allemand. Quand le champ a été labouré je l'ai ensemencé de blé partout, toujours parce que je croyais qu'il n'y avait qu'un Prussien d'enterré." ".

Les diagnostics archéologiques réalisés sur des grandes superficies permettent de constater souvent une surreprésentation de sépultures allemandes qui ne correspond pas à une mortalité plus importante mais bien à des négligences lors des opérations de transfert des corps. Les monuments funéraires, nombreux dans les cimetières allemands durant le conflit, ont fait l'objet de destructions au motif qu'ils étaient trop ostentatoires. De même, les tombes de soldats allemands présentes dans les cimetières communaux ont été rapidement transférées, alors que pour celles des Alliés les procédures ont pris en compte l'avis des familles et des autorités compétentes pour chaque nationalité. L'aménagement des nécropoles de regroupement de sépultures allemandes par les services français illustre le peu de considération pour le sacrifice des soldats ennemis : utilisation maximale de l'espace, pas d'aménagement paysager, interdiction de dépôts de fleurs, uniformité des croix de couleur noire [ill. 10]. Il a fallu attendre un deuxième conflit et les accords de réconciliation de 1966 pour que les Allemands retrouvent le droit de réorganiser et d'entretenir les nécropoles où reposent leurs soldats sur le territoire français.

Les différentes nations belligérantes venues se battre sur le front occidental ont été amenées à gérer des morts du camp adverse. On n'observe pas durant la Grande Guerre de pratiques généralisées d'acharnement sur les cadavres des ennemis. Certes, les Français ne semblent pas avoir porté une grande considération aux dépouilles des soldats allemands, mais les comportements discriminants se sont limités à des inhumations en sépulture multiple un peu plus fréquentes et à un respect moindre de leur identité. De leur côté, les Allemands ont considéré les soldats ennemis morts dans l'honneur des combats comme étant dignes d'égards. Aussi les ont-ils inhumés aux côtés des leurs, au sein de leurs cimetières. Point de traces de la haine entre les belligérants dans les pratiques funéraires, même si on décèle éventuellement du mépris ou de l'indifférence dont on ne sait si elle n'est pas simplement due à la mort de masse. En revanche, à l'issue de la guerre, les vainqueurs, appliquant le principe du vae victis, n'ont pas fait preuve de bonne volonté quant à la gestion des sépultures de guerre de leurs ennemis. 


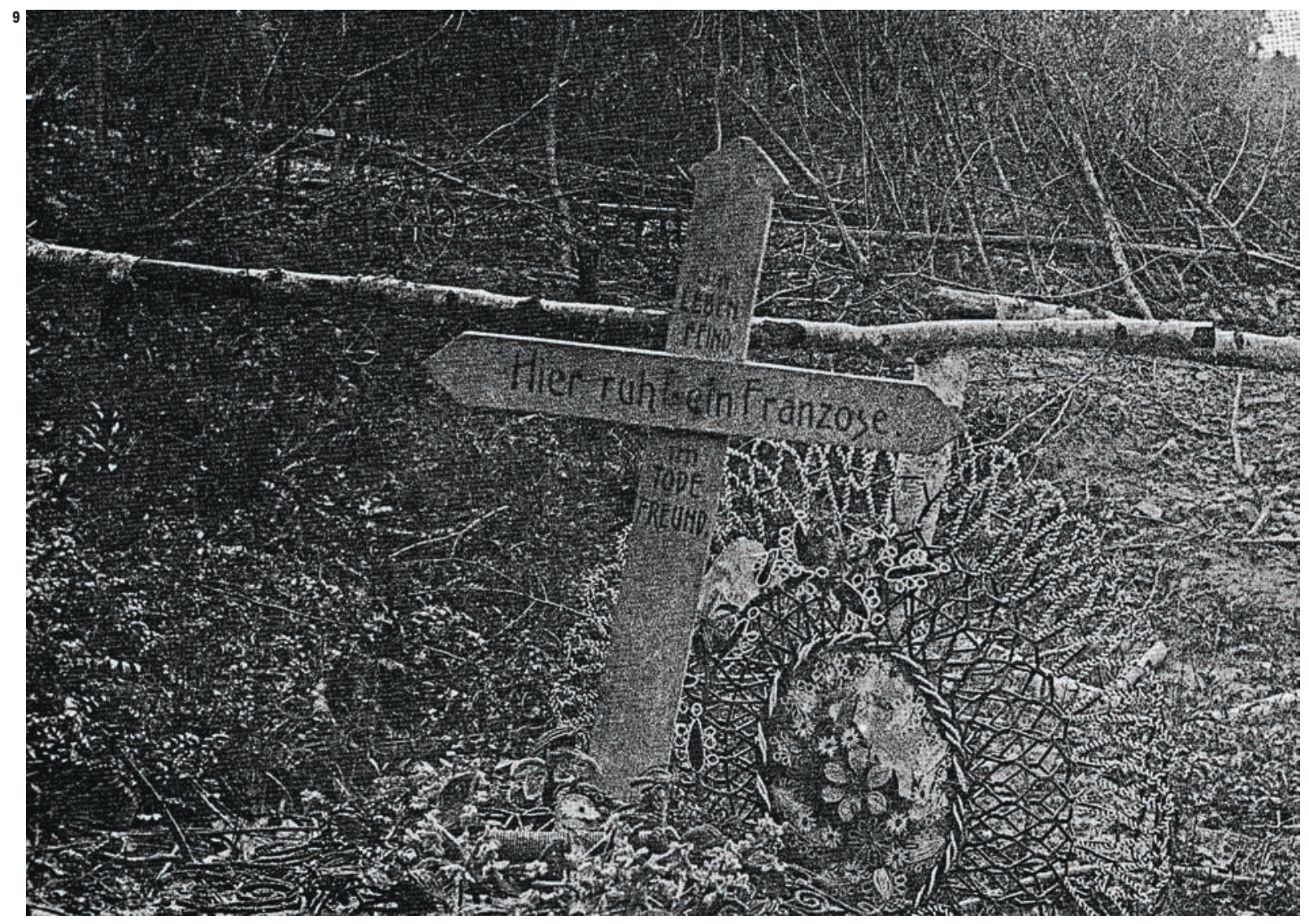

9. Cette photographie

d'une sépulture de soldat

français aménagée

par les Allemands (lieu

inconnu) illustre

un phénomène assez peu

répandu d'hommage

rendu au soldat ennemi :

« Hier ruh ein Franzose,

In Leben Feind im Tode

Freund - Ici repose un

Français, ennemi dans

la vie ami dans la mort ॥.

10. À partir de 1919,

un cimetière de

regroupement

de sépultures allemandes

est aménagé par

les Français à Sissonne

(Aisne) on constate

le peu d'égards accordés

aux sépultures

des ennemis vaincus.

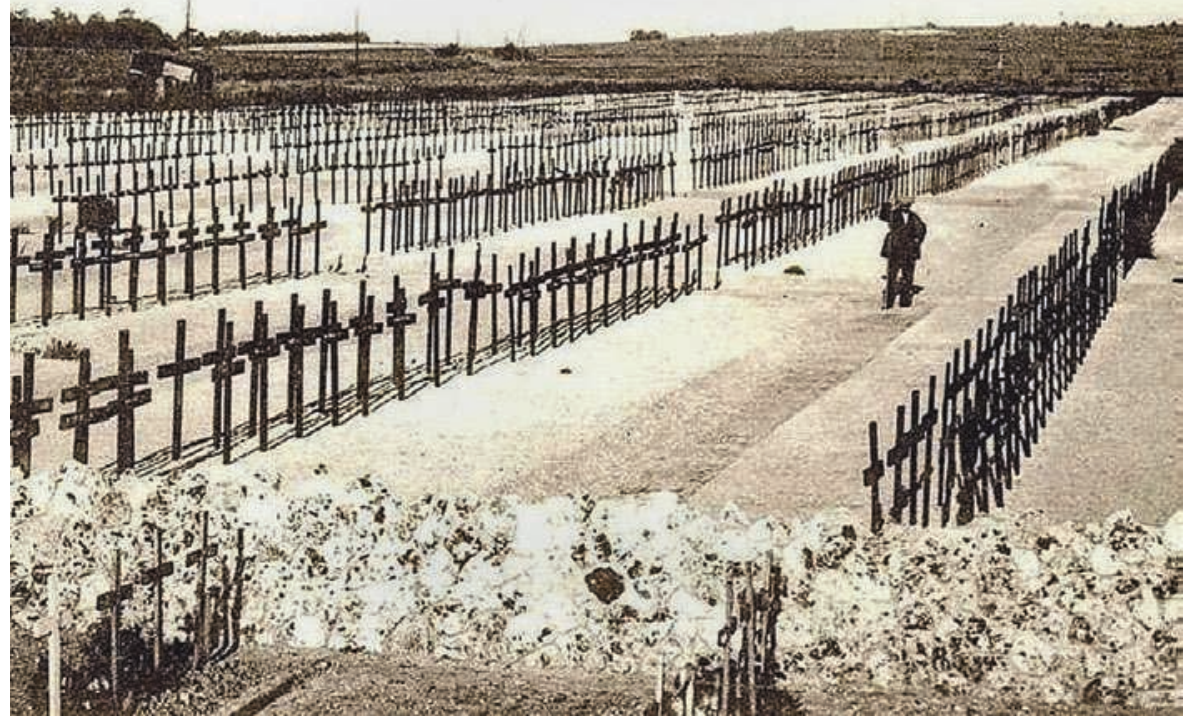

Références bibliographiques

BARTHAS L., 1987, Les carnets de guerre de Louis Barthas tonnelier, 1914-1918, Paris, La Découverte, 553 p.

Cazals R. (éd.), 2008, 1914-1918. Images de l'arrièrefront. Raoul Berthelé, lieutenant et photographe, Toulouse, Privat, $127 \mathrm{p}$

JüngER E., 2014, Carnets de guerre 1914-1918, Paris, Christian Bourgeois, $496 \mathrm{p}$

PotTeCHer I., 2003, 1914-1918, Lettres d'un fils. Un infirmier de chasseurs à pied à Verdun et dans l'Aisne, Louviers, Ysec, $208 \mathrm{p}$.

Pousse A., 200o, Une soutane sous la mitraille. Carnets de la Grande Guerre d'un curé de campagne, Jaignes, La Chasse au Snark, $196 \mathrm{p}$
Retailleau L., 2003, Musicien-brancardier. Carnets de Léopold Retailleau du $77^{e}$ R.I., 1914-1948,

Retailleau C. et Labayle É. (éd.), Parçay-surVienne, Anovi, 544 p.

Vuillermet F. A., 1918, Avec les Alpins. Impressions d'un prêtre-soldat, Paris, Lethielleux, $217 \mathrm{p}$.

Audouin-Rouzeau S., 2001, Cinq deuils de guerre 1914-1918, Paris, Éditions Noésis, 260 p.

Capdevila L., Voldman D., 2002, Nos morts, les sociétés occidentales face aux tués de la guerre, Paris, Payot, $282 \mathrm{p}$

Desfossés Y., Jacques A., Prilaux G., 2008, L'archéologie de la Grande Guerre, Rennes, Éd. Ouest-France, $127 \mathrm{p}$.

Dutour O., 2002, « Traces de vies disparues, l'anthropologue face aux charniers ", SocioAnthropologie, $\mathrm{n}^{\circ}$ 12, Traces ; http://socioanthropologie.revues.org/146

FluCher G., 2011, Le Chemin-des-Dames, du champ d'honneur au champ des morts, Louviers, Ysec, 127 p.

HARDIER T. ET JAGIELSKI J.-F, 2001, Combattre et mourir pendant la Grande Guerre (1914-1925) Paris, Imago, $375 \mathrm{p}$

JEAUFFRET J.-C., 1997, «La question du transfert des corps : 1915-1934 ", in CAzAls R. (dir.), Traces de 1914-1918, Carcassonne, Les Audois, p. 133-146.

Pourcher Y., 1993, « La fouille des champs d'honneur. La sépulture des soldats de 14-18 ", Terrain, $\mathrm{n}^{\circ} 20$ mars 1993, p. 37-56

SignOLI M., 2008, « Archéo-anthropologie funéraire et épidémiologique ", Socio-anthropologie, 22 ; http://socio-anthropologie.revues.org/115

Thomas L.-V., 1975, Anthropologie de la mort, Paris, Payot, $540 \mathrm{p}$

Urbain J-D 1998, L'archipel des morts. Cimetières et mémoires en Occident, Paris, Payot et Rivages, $357 \mathrm{p}$ 